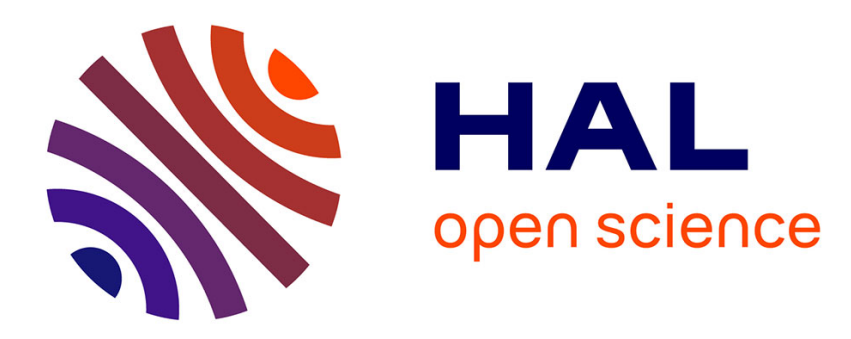

\title{
Directional effect of a magnetic field on oscillatory low-Prandtl-number convection
}

\author{
Daniel Henry, Anne Juel, Hamda Ben Hadid, Slim Kaddeche
}

\section{To cite this version:}

Daniel Henry, Anne Juel, Hamda Ben Hadid, Slim Kaddeche. Directional effect of a magnetic field on oscillatory low-Prandtl-number convection. Physics of Fluids, 2008, 20, pp.034104. 10.1063/1.2856125. hal-00442586

\section{HAL Id: hal-00442586 \\ https://hal.science/hal-00442586}

Submitted on 14 Jun 2012

HAL is a multi-disciplinary open access archive for the deposit and dissemination of scientific research documents, whether they are published or not. The documents may come from teaching and research institutions in France or abroad, or from public or private research centers.
L'archive ouverte pluridisciplinaire HAL, est destinée au dépôt et à la diffusion de documents scientifiques de niveau recherche, publiés ou non, émanant des établissements d'enseignement et de recherche français ou étrangers, des laboratoires publics ou privés. 


\title{
Directional effect of a magnetic field on oscillatory low-Prandtl-number convection
}

\author{
D. Henry, ${ }^{1}$ A. Juel, ${ }^{2}$ H. Ben Hadid, ${ }^{1}$ and S. Kaddeche ${ }^{3}$ \\ ${ }^{1}$ Laboratoire de Mécanique des Fluides et d'Acoustique, CNRS/Université de Lyon, \\ École Centrale de Lyon/Université Lyon 1/INSA de Lyon, ECL, 36 Avenue Guy de Collongue, \\ 69134 Ecully Cedex, France \\ ${ }^{2}$ Manchester Centre for Nonlinear Dynamics and School of Mathematics, \\ The University of Manchester, Oxford Road, Manchester M13 9PL, United Kingdom \\ ${ }^{3}$ Institut National des Sciences Appliquées et de Technologie, Unité de Recherche Matériaux, \\ Mesures et Applications, INSAT, B.P. 676, 1080 Tunis Cedex, Tunisia
}

(Received 11 June 2007; accepted 29 November 2007; published online 7 March 2008)

\begin{abstract}
The directional effect of a magnetic field on the onset of oscillatory convection is studied numerically in a confined three-dimensional cavity of relative dimensions 4:2:1 (length:width:height) filled with mercury and subject to a horizontal temperature gradient. The magnetic field suppresses the oscillations most effectively when it is applied in the vertical direction, and is the least efficient when applied in the longitudinal direction (parallel to the temperature gradient). In all cases, however, exponential growths of the critical Grashof number, $\mathrm{Gr}_{c}$ (Gr, ratio of buoyancy to viscous dissipation forces) with the Hartmann number (Ha, ratio of magnetic to viscous dissipation forces) are obtained. Insight into the damping mechanism is gained from the fluctuating kinetic energy budget associated with the time-periodic disturbances at threshold. The kinetic energy produced by the vertical shear of the longitudinal basic flow dominates the oscillatory transition, and when a magnetic field is applied, it increases in order to balance the stabilizing magnetic energy. Moreover, subtle changes in the spatial distribution of this shear energy are at the origin of the exponential growth of $\mathrm{Gr}_{c}$. The destabilizing effect of the velocity fluctuations strongly decreases when $\mathrm{Ha}$ is increased (due to the decay of the velocity fluctuations in the bulk accompanied by the appearance of steep gradients localized in the Hartmann layers), so that an increase of the shear of the basic flow at $\mathrm{Gr}_{c}$ is required in order to sustain the instability. This yields an increase in $\mathrm{Gr}_{c}$, which is reinforced by the fact that the shear of the basic flow naturally decreases at constant $\mathrm{Gr}$ with the increase of $\mathrm{Ha}$, particularly when the magnetic field is applied in the vertical direction. For transverse and longitudinal fields, the decay of the velocity fluctuations is combined with an increase of the shear energy term due to a sustained growth in stabilizing magnetic energy with Ha. (O) 2008 American Institute of Physics. [DOI: 10.1063/1.2856125]
\end{abstract}

\section{INTRODUCTION}

Directional solidification is used in the processing of semiconducting and optoelectronic materials, whose performance relies on the homogeneity of the crystalline material. ${ }^{1}$ In the horizontal Bridgman technique, the molten crystal is contained in a crucible which is withdrawn horizontally from a furnace. Thus, the melt is subject to a horizontal temperature gradient, which drives endwall convection. In practice, instabilities in the melt-phase adversely affect the quality of the crystal, as they impose temperature-fluctuations at the solidification front and lead to striations in the crystalline product. $^{2}$ The application of a magnetic field is common in modern crystal growing facilities because of its overall damping effect on the convective flow. In particular, striations may be eliminated by choosing a suitable magnetic field, as shown independently by Utech and Fleming ${ }^{3}$ and Hurle. $^{4}$

Thus, there is considerable interest in understanding the damping action of the magnetic field on time-dependent endwall convection in molten metals. The melts are typically excellent thermal conductors so that the Prandtl number (ra- tio of viscous to thermal diffusivity) is of the order of $10^{-2}$. The other parameters governing the magnetohydrodynamic convective flow are the Grashof number (ratio of buoyancy to viscous diffusion forces) and the Hartmann number (ratio of Lorentz to viscous diffusion forces).

The influence of a magnetic field on oscillatory convection in a horizontal Bridgman geometry was first addressed experimentally by Hurle et al. ${ }^{5}$ They considered a transverse magnetic field (perpendicular to both gravity and the applied temperature gradient) and found that the critical Grashof number for the onset of time-periodic convection, $\mathrm{Gr}_{c}$, followed a $\mathrm{Ha}^{2}$ dependence, which indicates the damping of oscillations with increasing magnetic field. A later study with the same experimental apparatus ${ }^{6}$ revealed chaotic dynamics for supercritical values of Ha. In strongly time-dependent convective flows in a vertical slot, temperature fluctuations were enhanced under weak, horizontal magnetic fields due to the formation of large scale convective structures. These were in turn suppressed with the increase of Ha. Recent experiments by Hof $e t a l .^{7}$ focused on the directional effect of the magnetic field in a rectangular enclosure of relative di- 
mensions 5.0:1.3:1.0 (length:width:height). They found that the critical Grashof number scales exponentially with Ha for the three principal orientations of the magnetic field. The magnetic field suppresses the oscillation most effectively when it is applied in the vertical direction $\left[\mathrm{Gr}_{c} / \mathrm{Gr}_{c}(\mathrm{Ha}=0)\right.$ $\left.\propto \exp \left(5.5 \times 10^{-3} \mathrm{Ha}^{3}\right)\right]$, compared with the transverse and longitudinal directions $\left[\mathrm{Gr}_{c} / \mathrm{Gr}_{c}(\mathrm{Ha}=0) \propto \exp (1.1\right.$ $\left.\times 10^{-2} \mathrm{Ha}^{2}\right)$ and $\mathrm{Gr}_{c} / \mathrm{Gr}_{c}(\mathrm{Ha}=0) \propto \exp \left(2.0 \times 10^{-3} \mathrm{Ha}^{2}\right)$, respectively]. The dependence of the frequency of the oscillations on Gr was found to be approximately similar to that measured in the absence of a magnetic field.

The exponential growth of $\mathrm{Gr}_{c}$ with $\mathrm{Ha}$ demonstrates the considerable effect of the magnetic field on the timedependent flow even for small values of $\mathrm{Ha}$, for which the modifications of the underlying bulk flow are usually considered as minimal compared to the strong modifications observed at large $\mathrm{Ha}^{8-12}$ In this paper, we provide insight into the damping mechanisms at play through the direct computation of Hopf bifurcation points and the analysis of the associated three-dimensional flow solutions. Similar continuation calculations have been performed in the absence of a magnetic field in a rectangular parallepiped enclosure, revealing multiple flow structures depending on the values of the aspect ratios and Prandtl number. ${ }^{13}$ Here, we choose an enclosure of dimensions 4:2:1 and a Prandtl number of $\mathrm{Pr}$ $=0.026$, for which the time-periodic flow at $\mathrm{Ha}=0$ is well understood and characterized. ${ }^{14}$ Coincidentally, the enclosure dimensions chosen by Hof et al. ${ }^{7}$ have been found to yield significant resolution issues. ${ }^{15}$

Most previous theoretical work on the magnetohydrodynamic damping of oscillatory convection has been focused on the linear stability analysis of convective flows in infinitely extended layers subject to a horizontal temperature gradient. ${ }^{16-19}$ When the horizontal confining plates are rigid, ${ }^{16}$ the vertical field is most effective at stabilizing the flow, suppressing both two-dimensional steady instability modes $\left[\mathrm{Gr}_{c} / \mathrm{Gr}_{c}(\mathrm{Ha}=0) \sim \exp \left(\mathrm{Ha}^{2}\right)\right]$ and three-dimensional oscillatory modes $\left[\mathrm{Gr}_{c} / \mathrm{Gr}_{c}(\mathrm{Ha}=0)-1 \sim \mathrm{Ha}^{2}\right]$. The strong stabilization of the two-dimensional modes correlates with a similar reduction in the shear energy normalized by $\mathrm{Gr}_{c}$. The horizontal directions of the field are significantly less effective at damping instabilities, with the transverse and longitudinal field each acting only on three-dimensional and twodimensional modes, respectively. Qualitatively similar results are found in the case of a free upper surface, ${ }^{17}$ while the effect of vertical and horizontal magnetic fields on the stability of thermocapillary convective flows has been addressed numerically by Priede and Gerbeth. ${ }^{20,21}$

The stability of endwall convection in a two-dimensional channel with an aspect ratio (length/height) of 4 has been established by means of Galerkin simulations. ${ }^{22}$ The vertical orientation of the magnetic field was most efficient at postponing the onset of oscillations to higher values of $\mathrm{Gr}_{c}$, whereas the longitudinal field was the least efficient, consistently with the experimental findings of Hof et al. ${ }^{7}$ The fact that several different oscillatory modes were encountered at onset, however, and that the critical Grashof number was found to depend nonmonotonically on Ha, giving rise to hysteresis phenomena, indicates significant deviations from the

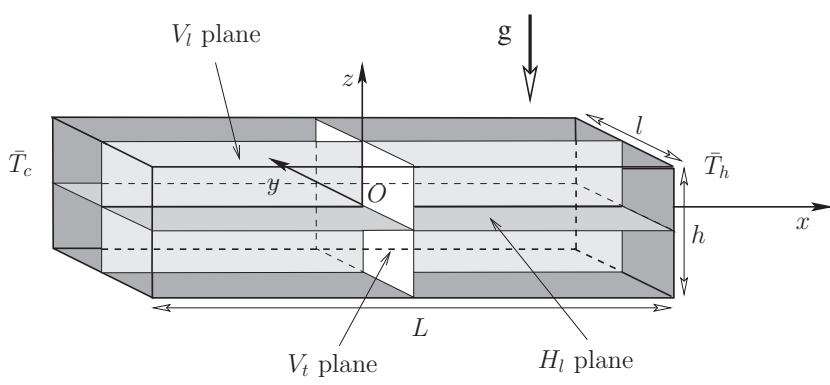

FIG. 1. Schematic diagram of the geometry of the differentially heated cavity.

experimental results. This is not surprising given the threedimensional nature of the bulk convective flow in enclosures of moderate lateral extent. ${ }^{12,23}$ By performing threedimensional continuation calculations of magnetohydrodynamic convection for the three principal directions of the magnetic field, we find monotonic Hopf bifurcation curves, where $\mathrm{Gr}_{c}$ depends exponentially on $\mathrm{Ha}$ as in the experiments of Hof et al. ${ }^{7}$ We shed light on the damping mechanisms involved with the analysis at marginal stability of the fluctuating kinetic energy budget associated with timeperiodic disturbances.

\section{MATHEMATICAL FORMULATION AND NUMERICAL METHOD}

The mathematical model consists of a differentially heated, rectangular parallelepiped cavity filled with an electrically conducting low-Pr fluid and placed in a constant magnetic field. The cavity has aspect ratios $A_{x}=L / h$ and $A_{y}$ $=l / h$, where $L$ is the length of the cavity (along $x$ ), $h$ its height (along $z$ ) and $l$ its width (along $y$ ), as shown schematically in Fig. 1. The vertical endwalls are isothermal and held at different temperatures, $\bar{T}_{h}$ at the right hot endwall and $\bar{T}_{c}$ at the left cold endwall, resulting in a horizontal applied temperature gradient. The sidewalls are adiabatic and all the walls are electrically insulating. The fluid is assumed to be Newtonian with constant physical properties (kinematic viscosity $\nu$, thermal diffusivity $\kappa$, density $\rho$ ), except for the density in the buoyancy term, which in the Boussinesq approximation, depends linearly on temperature, $\rho=\rho_{m}[1$ $\left.-\beta\left(\bar{T}-\bar{T}_{m}\right)\right]$, where $\beta$ is the thermal expansion coefficient, $\bar{T}_{m}$ is the mean temperature, $\bar{T}_{m}=\left(\bar{T}_{h}+\bar{T}_{c}\right) / 2$, and $\rho_{m}$ is the value of the density at $\bar{T}_{m}$. Moreau ${ }^{24}$ has shown that in most laboratory experiments using molten metals, the induced magnetic field is negligible, so that the applied magnetic field, $\boldsymbol{B}=|B| \boldsymbol{e}_{B}$, can be considered as the effective magnetic field. Thus, the convective motion is governed by the Navier-Stokes equations coupled to an energy equation. Using $h, h^{2} / \nu, \nu / h, \rho_{m} \nu^{2} / h^{2}, \gamma=\left(\bar{T}_{h}-\bar{T}_{c}\right) / A_{x}, \nu|B|$ and $\sigma_{e} \nu|B| / h\left(\sigma_{e}\right.$ is the electric conductivity) as scales for the length, time, velocity, pressure, temperature, induced electric potential and induced current respectively, these equations take the following form:

$\boldsymbol{\nabla} \cdot \boldsymbol{u}=0$ 


$$
\begin{aligned}
& \frac{\partial \boldsymbol{u}}{\partial t}+(\boldsymbol{u} \cdot \boldsymbol{\nabla}) \boldsymbol{u}=-\boldsymbol{\nabla} p+\boldsymbol{\nabla}^{2} \boldsymbol{u}+\mathrm{GrTe}_{z}+\mathrm{Ha}^{2} \boldsymbol{j} \times \boldsymbol{e}_{B}, \\
& \frac{\partial T}{\partial t}+(\boldsymbol{u} \cdot \boldsymbol{\nabla}) T=\frac{1}{\operatorname{Pr}} \nabla^{2} T .
\end{aligned}
$$

The dimensionless variables are the velocity vector $\boldsymbol{u}$ $=(u, v, w)$, the pressure $p$, the temperature $T=\left(\bar{T}-\bar{T}_{m}\right) / \gamma$, and the induced electric current density $j$. The nondimensional parameters arising from the scaling of the equations are the Grashof number, $\mathrm{Gr}=\beta g \gamma h^{3} / \nu^{2}$, the Prandtl number, $\operatorname{Pr}$ $=\nu / \kappa$ and the Hartmann number $\mathrm{Ha}=|B| h \sqrt{\sigma_{e} / \nu \rho_{m}} . \boldsymbol{e}_{z}$ and $\boldsymbol{e}_{B}$ are unit vectors in the vertical direction and in the direction of $\boldsymbol{B}$, respectively. In the equation of motion (2), the body force $\mathrm{Ha}^{2} \boldsymbol{j} \times \boldsymbol{e}_{B}$ is the Lorentz force, which results from the interaction between the induced electric current density $\boldsymbol{j}$ and the applied magnetic field $\boldsymbol{B}$. The dimensionless electric current density $\boldsymbol{j}$ is given by Ohm's law for a moving fluid,

$$
j=-\nabla \phi+u \times e_{B}
$$

where $\phi$ is the dimensionless electric potential. Combining the continuity equation for $\boldsymbol{j}, \boldsymbol{\nabla} \cdot \boldsymbol{j}=0$, and Ohm's law (4), we obtain the dimensionless equation governing the electric potential,

$$
\nabla^{2} \phi=e_{B} \cdot(\nabla \times u) .
$$

The boundary conditions are given by $\partial T / \partial z=0$ on $z$ $= \pm 1 / 2$ and $\partial T / \partial y=0$ on $y= \pm A_{y} / 2, \quad T=-A_{x} / 2$ on $x=-A_{x} / 2$ and $T=A_{x} / 2$ on $x=A_{x} / 2$, and $\boldsymbol{u}=0$ and $\partial \phi / \partial n=0$ on all boundaries.

In the Boussinesq approximation, the steady convective flow in this geometry exhibits two distinct symmetries for moderate $\mathrm{Gr},{ }^{14}$ a reflection symmetry $S_{l}$ with respect to the longitudinal $V_{l}$ plane (left-right symmetry) and a $\pi$-rotational symmetry $S_{r}$ about the transverse $y$-axis. These symmetries are defined, respectively, as

$S_{l}:(x, y, z, t) \rightarrow(x,-y, z, t), \quad(u, v, w, T) \rightarrow(u,-v, w, T)$,

$S_{r}:(x, y, z, t) \rightarrow(-x, y,-z, t), \quad(u, v, w, T) \rightarrow(-u, v,-w,-T)$.

The combination of these two symmetries yields a symmetry $S_{c}$ with respect to the center point of the cavity $\left(S_{c}=S_{l} \cdot S_{r}\right)$. When increasing Gr, bifurcations to new flow states (steady or oscillatory) will occur, at which some of these symmetries will usually be broken.

Equations (1)-(5) coupled to the boundary conditions were solved in a three-dimensional domain using a spectral element method described by Karniadakis et al. ${ }^{25}$ The time discretization was carried out using a semi-implicit splitting scheme where the nonlinear terms were first integrated explicitly, the pressure was then solved through a pressure equation enforcing the incompressibility constraint (with a consistent pressure boundary condition derived from the equations of motion), and the linear terms were finally integrated implicitly. This time-integration scheme was used for transient computations with the third-order accurate formulation described in Karniadakis et al. ${ }^{25}$
TABLE I. Mesh refinement tests of numerical accuracy of the critical Grashof number $\mathrm{Gr}_{c}$ for the onset of time-periodic convection in a laterally heated three-dimensional cavity $\left(A_{x}=4, A_{y}=2\right.$, and $\left.\operatorname{Pr}=0.026\right)$ : (a) without magnetic field $(\mathrm{Ha}=0)$, (b) with a vertical magnetic field $(\mathrm{Ha}=8.3)$, (c) with a transverse magnetic field $(\mathrm{Ha}=21)$, (d) with a longitudinal magnetic field $(\mathrm{Ha}=43.5)$. In cases (b)-(d), the value of $\mathrm{Ha}$ is the highest value used in the calculations for the given direction of the magnetic field.

\begin{tabular}{lccc}
\hline \hline Mesh & $43 \times 45 \times 23$ & $47 \times 49 \times 27$ & $51 \times 53 \times 31$ \\
\hline (a) & 32728.8 & 32726.9 & 32726.6 \\
(b) & 213794 & 213273 & 212746 \\
(c) & 217367 & 217301 & 217395 \\
(d) & 233615 & 233618 & 233646 \\
\hline \hline
\end{tabular}

The same refined mesh comprising $47 \times 49 \times 27$ points (in the $x, y$, and $z$ directions, respectively) was chosen for all our calculations of convective flow in a cavity of aspect ratios $A_{x}=4$ and $A_{y}=2$, subject to a magnetic field of varying direction and magnitude. As shown by the convergence tests given in Table I, this mesh yields excellent resolution of the threshold, $\mathrm{Gr}_{c}$, in the absence of a magnetic field. The precision slightly decreases when the intensity of the applied magnetic field is increased, but it remains satisfactory even for the largest values of $\mathrm{Ha}$ (Table I). The least accurate results are obtained for a vertical magnetic field at $\mathrm{Ha}=8.3$ (the highest value of Ha used for this field direction). In this case, the variation of $\mathrm{Gr}_{c}$ with Ha is very steep, but the value of $\mathrm{Gr}_{c}$ changes by less than $0.25 \%$ when the mesh is further resolved.

We focused on following steady flow solutions by incrementing $\mathrm{Gr}$, and locating bifurcation points at a critical value of the Grashof number, $\mathrm{Gr}_{c}$. The Newton method described by Henry and Ben Hadid ${ }^{13}$ was used to calculate each steady state solution. Leading eigenvalues and their corresponding eigenvectors were then determined using Arnoldi's method $\left(\right.$ ARPACK library $^{26}$ ) by time-stepping the linearized equations, as described by Mamun and Tuckerman. ${ }^{27}$ The real parts of the leading eigenvalues were monitored in order to locate the bifurcation point approximately (i.e., the largest value of $\mathrm{Gr}$ for which the real part of the leading eigenvalue remained negative). The steady solution and the leading eigenvectors corresponding to this estimated threshold were in turn used as initial guesses in the direct calculation of the bifurcation point, which was performed using the Newton method described by Petrone et al. ${ }^{28}$ and Henry and Ben Hadid. ${ }^{13}$ In the Newton methods used for both steady state solving and threshold calculations, the main idea was to solve the linear systems appearing at each Newton step with an iterative solver, and to compute right-hand sides and matrix-vector products corresponding to these linear systems by performing adapted first order time steps of the basic or linearized problem. The advantage of this method was that the Jacobian matrix did not need to be constructed or stored. The GMRES algorithm from the NSPCG (Ref. 29) software library was used as the iterative solver.

Important information concerning the physical mechanisms involved in the transition to the oscillatory state and in the stabilization by the applied magnetic field can be 
obtained from the calculation at threshold of the fluctuating kinetic energy budget associated with time-periodic disturbances. The basic steady solution at threshold $[u, v, w, T](x, y, z)\left\{\right.$ or $\left.\left[u_{i}, T\right]\left(x_{i}\right)\right\}$ and the complex critical eigenvector $\left[u^{\prime}, v^{\prime}, w^{\prime}, T^{\prime}\right](x, y, z)$ or $\left.\left[u_{i}^{\prime}, T^{\prime}\right]\left(x_{i}\right)\right\}$ both enter the equation of energy budget given by

$$
\frac{\partial k}{\partial t}=e_{\mathrm{shear}}+e_{\mathrm{visc}}+e_{\mathrm{buoy}}+e_{\mathrm{magn}}+e_{\mathrm{pres}}
$$

where $\partial k / \partial t$ is the rate of change of the fluctuating kinetic energy defined as $k=\mathcal{R} e\left(u_{i}^{\prime} u_{i}^{\prime *} / 2\right)(\mathcal{R} e$ and the superscript $*$ denoting the real part and the complex conjugate, respectively), and

$$
\begin{aligned}
& e_{\text {shear }}=\mathcal{R} e\left(-u_{j}^{\prime} \frac{\partial u_{i}}{\partial x_{j}} u_{i}^{\prime *}\right), \\
& e_{\text {visc }}=\mathcal{R} e\left(-\frac{\partial u_{i}^{\prime}}{\partial x_{j}} \frac{\partial u_{i}^{\prime *}}{\partial x_{j}}\right), \\
& e_{\text {buoy }}=\mathcal{R} e\left(\mathrm{Gr}^{\prime} u_{i}^{\prime *} \delta_{i 3}\right), \\
& e_{\text {magn }}=\mathcal{R} e\left[\mathrm{Ha}^{2}\left(\boldsymbol{j}^{\prime} \times \boldsymbol{e}_{B}\right) \boldsymbol{u}^{\prime *}\right], \\
& e_{\text {pres }}=\mathcal{R} e\left(-\frac{\partial p^{\prime}}{\partial x_{i}} u_{i}^{\prime *}\right) .
\end{aligned}
$$

$e_{\text {shear }}$ represents the production of fluctuating kinetic energy by shear of the basic flow, $e_{\mathrm{visc}}$ the viscous dissipation of fluctuating kinetic energy, $e_{\text {buoy }}$ the production of fluctuating kinetic energy by buoyancy, $e_{\text {magn }}$ the dissipation of fluctuating kinetic energy by the magnetic forces, and $e_{\text {pres }}$ the redistribution of fluctuating kinetic energy by the pressure fluctuations. We can also define the total (or volume integral) fluctuating kinetic energy as $K=\int_{\Omega} k d \Omega$. The rate of change of $K$ is given by an equation similar to Eq. (6), which involves the volume integral energy terms (denoted by $E$ ),

$$
\frac{\partial K}{\partial t}=E_{\mathrm{shear}}+E_{\mathrm{visc}}+E_{\mathrm{buoy}}+E_{\mathrm{magn}} .
$$

Note that the volume integral pressure term is zero and has therefore not been included in Eq. (7). At threshold, the critical eigenvector is associated with an eigenvalue of zero real part. This implies that $\partial k / \partial t$ and $\partial K / \partial t$ are both equal to zero at marginal stability. The calculation of all the individual energy contributions enables us to determine which term plays a dominant role in triggering the instability through production of fluctuating kinetic energy. The corresponding spatial fields $e(x, y, z)$ can in turn be analyzed to locate the production regions. Note that, as shown by Kaddeche et al., ${ }^{16} E_{\mathrm{visc}}$ and $E_{\mathrm{magn}}$ are stabilizing by nature and thus negative terms.

Finally, if we normalize Eq. (7) by $-E_{\text {visc }}=\left|E_{\text {visc }}\right|$, which is always positive, we can get another equation involving normalized energy terms $E^{\prime}=E /\left|E_{\mathrm{visc}}\right|$ at threshold,

$$
E_{\text {shear }}^{\prime}+E_{\text {buoy }}^{\prime}+E_{\text {magn }}^{\prime}=1 .
$$

Positive (negative) energy terms are destabilizing (stabilizing), respectively. In the remainder of the paper, we simplify the discussion of the damping mechanism by referring to the growth or decay of the absolute values of each energy term.

\section{RESULTS}

We focus on the transition to oscillatory flow in our model and explore the directional effect of a magnetic field on this transition, by applying the magnetic field in the three principal directions (vertical, i.e., parallel to gravity; transverse, i.e., perpendicular to gravity and to the imposed temperature gradient; and longitudinal, i.e., perpendicular to gravity and parallel to the imposed temperature gradient). We choose a cavity of aspect ratios $A_{x}=4$ and $A_{y}=2$, as the onset of time-dependent flow in this geometry has already been thoroughly studied by Henry and Buffat ${ }^{14}$ in the absence of a magnetic field. They characterize the flow transitions for several values of the Prandtl number, including $\operatorname{Pr}=0.026$ which corresponds to mercury. For $\mathrm{Pr}=0.026$, the increase of $\mathrm{Gr}$ leads to the concentration of the main convective circulation into a large roll in the core of the cavity. The oscillatory transition, which occurs through a Hopf bifurcation at a critical value of the Grashof number, $\mathrm{Gr}_{c}$, is accompanied by the breaking of the $S_{r}$ and $S_{l}$ symmetries, and results in a periodic flow, where the roll oscillates around the central point of the cavity. The analysis of the fluctuating kinetic energy budget close to threshold has shown that the main destabilizing contribution comes from shear, and more precisely from the term connected to the vertical gradient of the longitudinal velocity of the mean flow. Here, we choose to examine the influence of the magnetic field on this specific flow transition. Also, the shear term mentioned above has recently been shown to be responsible for the destabilization of convective flows in end-heated cavities over a wide range of aspect ratios and Prandtl number values, ${ }^{13}$ suggesting that the findings of our case study may extend to a broad range of parameters. In Sec. III A, we discuss the dependence of $\mathrm{Gr}_{c}$ on the Hartmann number, and calculate the global energy budgets in the three-dimensional cavity. In order to facilitate the understanding of the damping effect of the magnetic field in the three-dimensional cavity, we choose in Sec. III B to extend the analysis of the more academic case of a fluid layer of infinite lateral extent, confined between horizontal plates and subject to a horizontal temperature gradient, which is strongly stabilized in the presence of a vertical magnetic field. ${ }^{16}$ The spatial distribution of the fluctuating kinetic energy budget is subsequently analyzed for the magnetic-fielddelayed transition in the three-dimensional cavity in Sec. III C, based on the methods introduced in Sec. III B.

\section{A. Stability curves and energy budgets for the three-dimensional cavity}

Stability curves representing the dependence of $\mathrm{Gr}_{c}$ on Ha, are shown in Fig. 2(a) for the three principal directions of the magnetic field (vertical along $z$, transverse along $y$, and longitudinal along $x$ ). The Hopf bifurcation at $\mathrm{Ha}=0$ was 

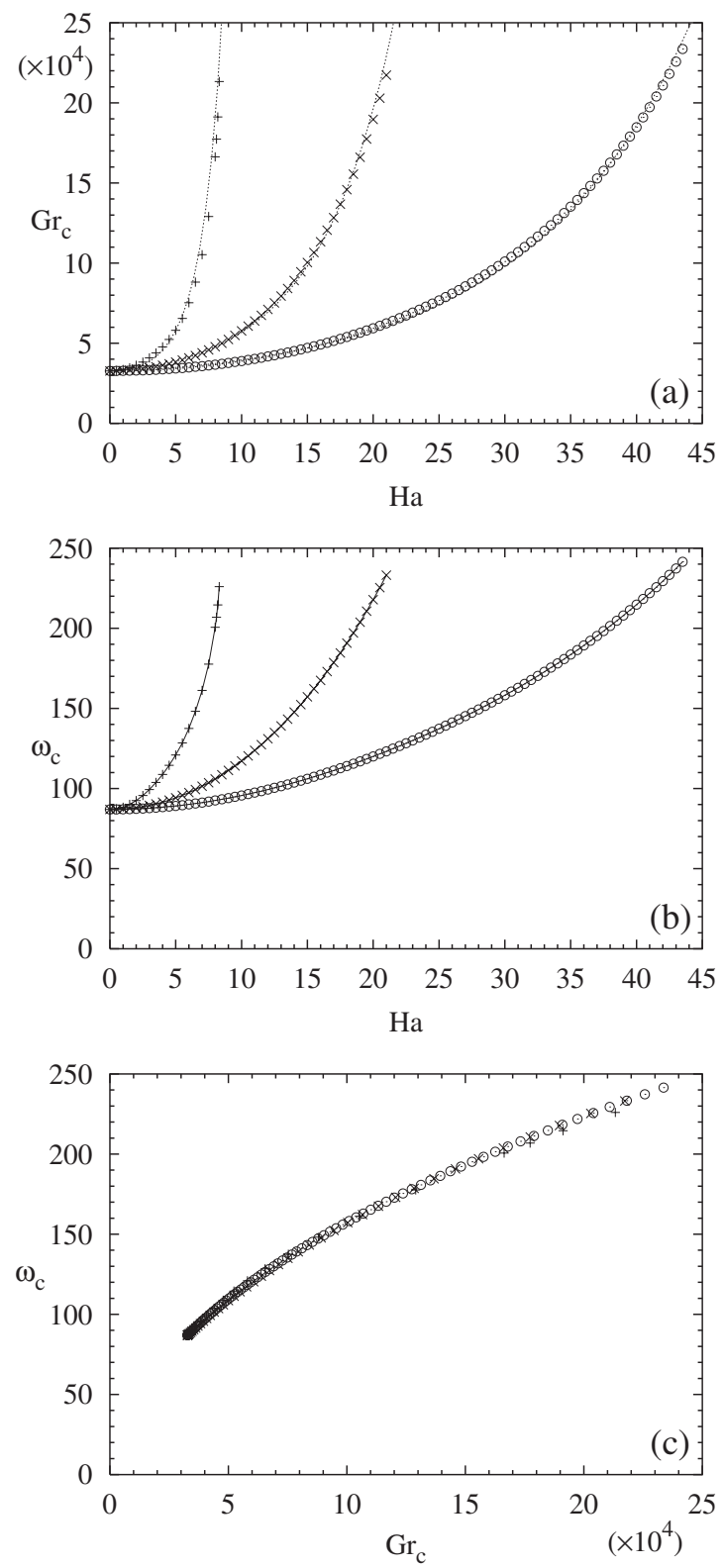

FIG. 2. Variation of the oscillatory threshold $\mathrm{Gr}_{c}$ (a) and of the corresponding angular frequency $\omega_{c}$ (b) as a function of Ha for a laterally heated three-dimensional cavity and three orientations of the magnetic field $(+$ for the vertical magnetic field, $\times$ for the transverse field, $\bigcirc$ for the longitudinal field). The dotted lines in (a) are the fits given in the text. The plot of the angular frequency as a function of $\mathrm{Gr}_{c}$ is shown in (c). Other parameters are $A_{x}=4, A_{y}=2$, and $\operatorname{Pr}=0.026$.

located first, using the method discussed in Sec. II. The threshold, $\mathrm{Gr}_{c}$, was computed directly by Newton's method, using previously calculated initial guesses for the bulk flow and the leading eigenvector. This direct method was subsequently employed to gradually obtain the Hopf bifurcation points for increasing values of $\mathrm{Ha}$ for each direction of the magnetic field. For each incremental value of $\mathrm{Ha}$, the steady solution and leading eigenvector determined at the previous step were used as initial guesses.

The steep monotonic growth of $\mathrm{Gr}_{c}$ with Ha shown in Fig. 2(a) demonstrates that all three directions of the magnetic field have a strong stabilizing influence. The dotted lines represent fits to the data for each direction of the mag- netic field of the form $\mathrm{Gr}_{c} / \mathrm{Gr}_{c}(\mathrm{Ha}=0) \propto \exp \left(a \mathrm{Ha}^{b}\right)$. Exponential fits were found to represent the numerical data most accurately over the entire range of $\mathrm{Ha}$ studied, compared with low order polynomial fits of the form $1+a \mathrm{Ha}^{b}$, which diverged from the data as $\mathrm{Ha}$ increased. Thus, in all three cases, the critical Grashof number exhibits an exponential dependence on powers of Ha, so that values of Ha of a few units are sufficient to double the threshold values. There are, however, significant differences in efficiency between the three directions of the magnetic field. The vertical magnetic field suppresses the oscillation most effectively with $a_{v}=12$ $\times 10^{-3}$ and $b_{v}=2.4$. Both the transverse and longitudinal fields are less effective than the vertical magnetic field at postponing the Hopf bifurcation, since the fits to the onset curves yield lower but approximately similar powers of $\mathrm{Ha}$ of $b_{t}=1.7$ and $b_{l}=1.6$. The action of a transverse field, however, results in significantly enhanced stabilization compared to that of the longitudinal field as $a_{t}=11 \times 10^{-3}$ is approximately 2.3 times larger than $a_{l}=4.75 \times 10^{-3}$. These findings are in qualitative agreement with the experimental results of Hof et al., ${ }^{7}$ who also measured exponential dependencies of $\mathrm{Gr}_{c}$ on powers of $\mathrm{Ha}$, and observed the strongest damping for the vertical magnetic field followed by the transverse and finally the longitudinal fields. The stabilization in our threedimensional model, however, is slightly weaker than in the experiment, with smaller exponents for the Ha dependence of the exponential, for each direction of the magnetic field. Note that the fits to the data are not expected to hold for any value of $\mathrm{Ha}$, due to the complete reorganization of the basic flow at high Hartmann numbers.

The dependence on Ha of the critical frequency of oscillation, $\omega_{c}$, is shown in Fig. 2(b). ( $\omega_{c}$ is the imaginary part of the leading eigenvalue at the Hopf bifurcation point.) The continuous nature of the curves indicates that the same mode of instability is retained over the range of Ha investigated for all three directions of the magnetic field, consistently with the experimental observations of Hof et al. ${ }^{7}$ This also points to important differences with the two-dimensional model of Gelfgat and Bar-Yoseph, ${ }^{22}$ who encountered multiple modes of oscillations. The functional dependence of the frequency on $\mathrm{Ha}$ is similar to that of the thresholds, thus yielding a stronger increase for the vertical magnetic field compared to the two other directions. Furthermore, when the critical frequency is plotted against $\mathrm{Gr}_{c}$ [see Fig. 2(c)], the curves corresponding to the different directions of magnetic field collapse, indicating that the growth of the critical frequency can be directly correlated to that of the threshold of the instability independently of the magnetic field. This result is closely linked to the observation by Hof et al. ${ }^{7}$ that the Grashof number dependence of the frequency of oscillation above onset is virtually independent of the magnetic field.

The four energy terms contributing to the rate of change of the total fluctuating kinetic energy at threshold [shear of the basic flow, buoyancy, viscous and magnetic dissipation, listed in Eq. (7)] were calculated from the basic flow solution and critical eigenvector. The shear term was decomposed into its nine individual contributions, corresponding to the gradients in each of the three directions of the three compo- 


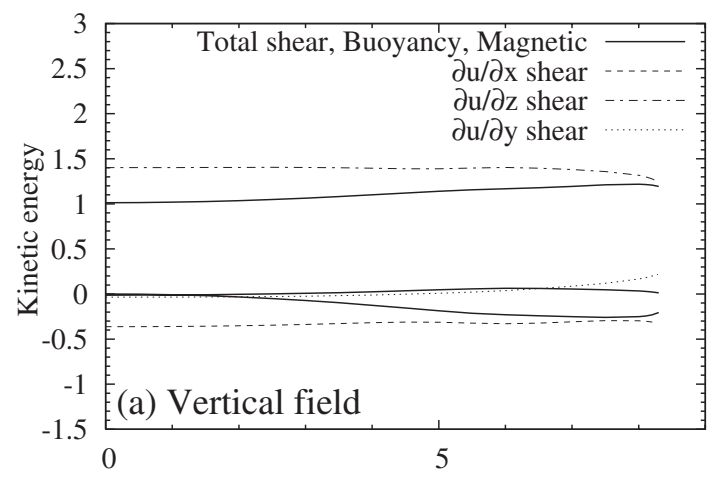

$\mathrm{Ha}$
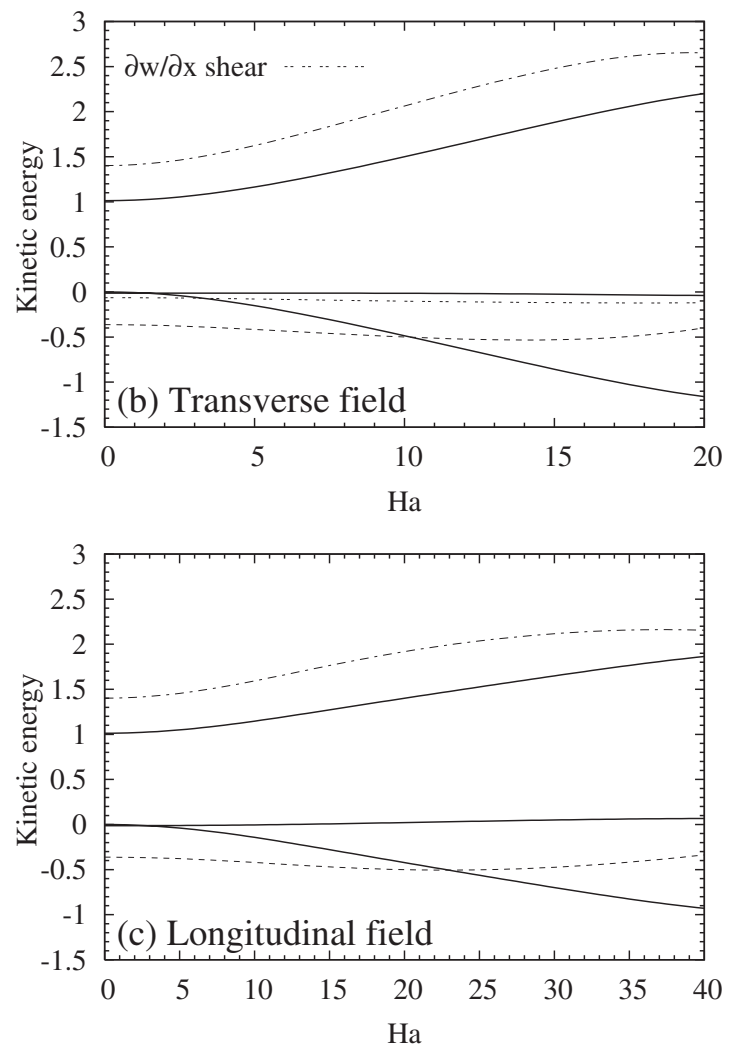

FIG. 3. Fluctuating kinetic energy budget associated with the oscillatory disturbances at threshold in a laterally heated three-dimensional cavity for the three orientations of the magnetic field: vertical (a), transverse (b), and longitudinal (c). The contributions, normalized by $\left|E_{\text {visc }}\right|$, are given as a function of Ha. Solid lines represent the total production by shear (increasing above 1), the magnetic dissipation (decreasing below 0), and the buoyancy contribution (around 0). Nonsolid lines represent the individual shear contributions, but only those larger than 0.1 in absolute value are given. Other parameters are $A_{x}=4, A_{y}=2$, and $\operatorname{Pr}=0.026$.

nents of the basic velocity, in order to identify the dominant ones. In addition, all the energy terms were normalized by $\left|E_{\text {visc }}\right|$ as expressed in Eq. (8).

The main individual energy contributions at threshold are shown as a function of Ha in Fig. 3 for the three orientations of the magnetic field. In all three cases, the dominant production of fluctuating kinetic energy is due to the shear of the basic flow. This destabilizing contribution is close to 1 for $\mathrm{Ha}=0$ as it acts to balance the viscous dissipation term, while the contribution of buoyancy is insignificant. As $\mathrm{Ha}$ is increased, the contribution of the destabilizing shear in- creases approximately proportionally to that of the stabilizing magnetic dissipation, while the buoyancy contribution remains negligibly small. These changes with increasing $\mathrm{Ha}$ are more pronounced for the transverse magnetic field than for the longitudinal field. Interestingly, they are weakest in the case of the vertical field, where the total shear and magnetic energy contributions exhibit only small growths before levelling off for values of Ha between 7.5 and 8, and then decreasing for larger values of Ha. Moreover, the decomposition of the shear term indicates that the production of fluctuating kinetic energy is essentially due to the strongly destabilizing term connected to $\partial u / \partial z$, whereas the term connected to $\partial u / \partial x$ is clearly stabilizing and all other terms are small in comparison, and thus negligible.

The above results demonstrate that the oscillatory transition is dominated by the shear of the basic flow, and more specifically by the vertical shear of the longitudinal velocity. This is the case both in the absence and in the presence of a magnetic field. When $\mathrm{Ha}>0$, the stabilizing magnetic contribution leads to the increase of both the total and dominant shear contributions. In the case of the vertical magnetic field, however, the stabilizing magnetic contribution rapidly levels off as Ha increases, despite the continued growth of the instability threshold. This suggests that the magnetic contribution is not the dominant source of stabilization in our flow configuration.

\section{B. Energy analysis of the transitions in a laterally heated layer subject to a vertical magnetic field}

We have seen in Sec. III A that the mechanisms responsible for the stabilization of the oscillatory flow in the presence of a magnetic field cannot simply be inferred from the analysis of the global energy budget. Thus, a detailed examination of the spatial distribution of the shear energy is necessary to gain insight into the damping action of the magnetic field. Our first approach is to consider the simpler problem of magnetohydrodynamic damping in an extended fluid layer confined between rigid, horizontal walls and subject to a horizontal temperature gradient, ${ }^{16}$ which presents important similarities with our three-dimensional problem and offers the advantage of an analytical basic flow solution. Indeed, the linear stability analysis of this basic flow yields a strong increase of the threshold for the two-dimensional steady instability, scaling as $\mathrm{Gr}_{c} / \mathrm{Gr}_{c}(\mathrm{Ha}=0) \sim \exp \left(\mathrm{Ha}^{2}\right)$. In addition, the analysis at threshold of the kinetic energy budget associated with the two-dimensional disturbances has shown that the dominant destabilizing contribution comes from the shear of the basic flow, and specifically the term connected to $(\partial u / \partial z)$, which incidentally is the only shear term in this simplified geometry.

An advantage of the analysis performed by Kaddeche et $a{ }^{16}{ }^{16}$ is that the basic flow $u(\mathrm{Gr}, \mathrm{Ha})$ is directly proportional to $\mathrm{Gr}, u(\mathrm{Gr}, \mathrm{Ha})=\mathrm{Gr} u_{G}(\mathrm{Ha})$. Thus, $\mathrm{Gr}$ can be factored out of the shear energy term, so that $E_{\text {shear }}^{\prime}=\mathrm{Gr} E_{\text {shear }}^{\prime \prime}$. A similar transformation applies to the energy due to buoyancy, which can be written as $E_{\text {buoy }}^{\prime}=\mathrm{Gr} E_{\text {buoy }}^{\prime \prime}$. Equation (8) at marginal stability can then be rewritten as 
TABLE II. Characterization of the stabilization by a vertical magnetic field for the two-dimensional steady disturbances developing in a laterally heated layer at $\operatorname{Pr}=0.001\left(\mathrm{Gr}_{c_{0}}=7943\right)$.

\begin{tabular}{lrrr}
\hline \hline $\mathrm{Ha}$ & $R_{1}$ & $R_{2}$ & $\mathrm{Gr}_{c} / \mathrm{Gr}_{c_{0}}$ \\
\hline 3 & 1.36 & 1.11 & 1.51 \\
5 & 2.25 & 1.28 & 2.88 \\
7 & 4.81 & 1.48 & 7.10 \\
9 & 14.79 & 1.55 & 22.88 \\
10 & 28.47 & 1.52 & 43.27 \\
11 & 56.54 & 1.48 & 83.48 \\
12 & 115.89 & 1.42 & 164.51 \\
13 & 239.76 & 1.36 & 325.99 \\
14 & 491.09 & 1.30 & 640.60 \\
\hline \hline
\end{tabular}

$$
\operatorname{Gr}_{c}\left(E_{\text {shear }}^{\prime \prime}+E_{\text {buoy }}^{\prime \prime}\right)=1-E_{\text {magn }}^{\prime},
$$

which leads to

$$
\frac{\mathrm{Gr}_{c}}{\mathrm{Gr}_{c_{0}}}=\overbrace{\left(\frac{E_{\text {shear }_{0}}^{\prime \prime}+E_{\text {buoy }_{0}}^{\prime \prime}}{E_{\text {shear }}^{\prime \prime}+E_{\text {buoy }}^{\prime \prime}}\right)}^{R_{1}} \overbrace{\left(1-E_{\text {magn }}^{\prime}\right.}^{R_{2}},
$$

where the values with the subscript 0 refer to the case where $\mathrm{Ha}=0$. Kaddeche et al. ${ }^{16}$ find that the strong increase of $\mathrm{Gr}_{c}$ with $\mathrm{Ha}$ is caused by the growth of the first factor $R_{1}$ (see Table II, where the results for $\operatorname{Pr}=0.001$ are reproduced). In this case, buoyancy is very weak, so that the growth of $\mathrm{Gr}_{c}$ is the consequence of the strong reduction in energy generated by shear through $E_{\text {shear, }}^{\prime \prime}$ when the vertical magnetic field is applied.

In order to deepen the analysis of the shear term $E_{\text {shear }}^{\prime \prime}$, we have recomputed the case corresponding to $\operatorname{Pr}=0.001$. The kinetic energy budget associated with two-dimensional disturbances is shown in Fig. 4 for $\mathrm{Ha}=0$ and $\mathrm{Ha}=14$, by plotting the individual energy contributions corresponding to those expressed in Eq. (6). Each energy term is normalized by $\left|E_{\text {visc }}\right|$, which yields $e^{\prime}$ terms. For $\mathrm{Ha}=0$ [Fig. 4(a)], the shear term is destabilizing in the central half of the layer. The viscous dissipation occurs in the regions adjacent to the walls, each of which extends to approximately a quarter of the depth of the layer, while the buoyancy term is very small everywhere. Thus, the pressure redistributes the energy of the disturbances from the center toward the walls. Significant changes occur when the magnetic field is applied [ $\mathrm{Ha}=14$, Fig. 4(b)]. The influence of the destabilizing shear extends over a broader region, while the viscous and small magnetic dissipations are concentrated in thin boundary layers (Hartmann layers), which develop along the walls.

The $E_{\text {shear }}^{\prime \prime}$ term is given by the integral across the layer of $e_{\text {shear }}^{\prime \prime}(z)$, which can itself be written as the product of two terms: $\left(-\partial u_{G} / \partial z\right)$, a quantity related to the analytical basic flow, which is independent of $\mathrm{Gr}_{c}$ and only dependent on Ha, and $\left[\mathcal{R} e\left(w^{\prime} u^{\prime *}\right) /\left|E_{\mathrm{visc}}\right|\right]$, a quantity related to the velocity disturbances at the threshold $\mathrm{Gr}_{c}$. Note that the quantities $\mathcal{R} e\left(w^{\prime} u^{\prime *}\right)$ and $\left|E_{\mathrm{visc}}\right|$ both depend on the normalization chosen for the critical eigenvector, due to the definition of the disturbances to within a multiplicative constant. Their ratio,
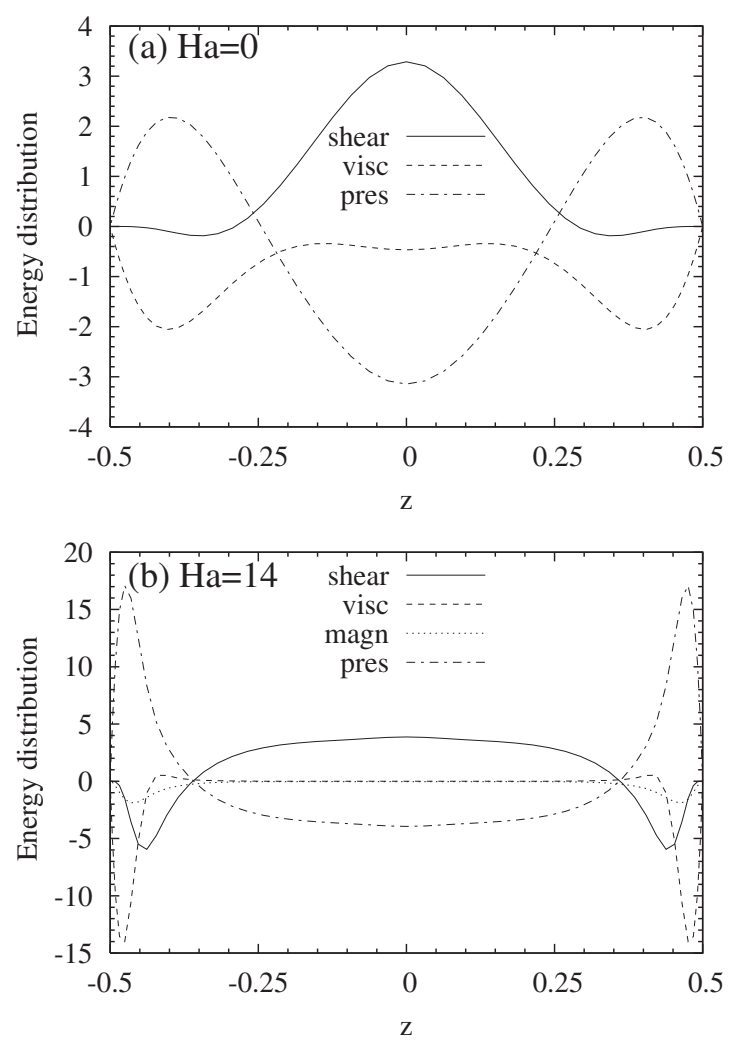

FIG. 4. Spatial distribution of the kinetic energy budget associated with the two-dimensional steady disturbances at threshold in a laterally heated layer at $\mathrm{Pr}=0.001$, without magnetic field $(\mathrm{Ha}=0)(\mathrm{a})$ and with a vertical magnetic field $(\mathrm{Ha}=14)(b)$. The buoyancy contribution which is small everywhere is not plotted.

$\left[\mathcal{R} e\left(w^{\prime} u^{\prime *}\right) /\left|E_{\mathrm{visc}}\right|\right]$, however, is independent of this normalization, and thus, this quantity is intrinsic to the flow perturbations. The z-profiles of these three quantities are plotted in Fig. 5 for increasing values of Ha. The most striking feature of these plots is the exponential decrease of $e_{\text {shear }}^{\prime \prime}$ as Ha is increased up to $\mathrm{Ha}=14$ [Fig. 5(a)], which drives the strong increase of the instability threshold. Note that the term $\left(-\partial u_{G} / \partial z\right)\left[\right.$ Fig. 5(b)] determines the sign of $e_{\text {shear, }}^{\prime \prime}$ since $\left[\mathcal{R} e\left(w^{\prime} u^{\prime *}\right) /\left|E_{\mathrm{visc}}\right|\right][$ Fig. $5(\mathrm{c})]$ is positive across the entire layer. Thus, the positive values of $\left(-\partial u_{G} / \partial z\right)$ found in the central part of the layer delimit the region of destabilization by shear. The maximum positive value of $e_{\text {shear }}^{\prime \prime}$ (corresponding to the most effective destabilization) is located at $z=0$, which also corresponds to the position of the inflection point of the basic velocity profile. Near the boundaries, however, $e_{\text {shear }}^{\prime \prime}$ takes small negative values, indicating a region of weak stabilization. As mentioned by Kaddeche et al., ${ }^{16}$ the strong decrease of $e_{\text {shear }}^{\prime \prime}$ is connected to a decrease of $\left(-\partial u_{G} / \partial z\right)$ in the central region, due to the flattening of the basic velocity profile around the inflection point induced by the vertical magnetic field [Fig. 5(b)]. The decrease of $e_{\text {shear }}^{\prime \prime}$, however, is dominated by the rapid decay of the velocity disturbances $\left[\mathcal{R} e\left(w^{\prime} u^{\prime *}\right) /\left|E_{\mathrm{visc}}\right|\right]$ shown in Fig. 5(c). Hence, the strong stabilization of the flow with increasing Ha results primarily from the efficient reduction of the scaled velocity disturbance product, rather than the modification of the basic velocity profile by the magnetic field. 

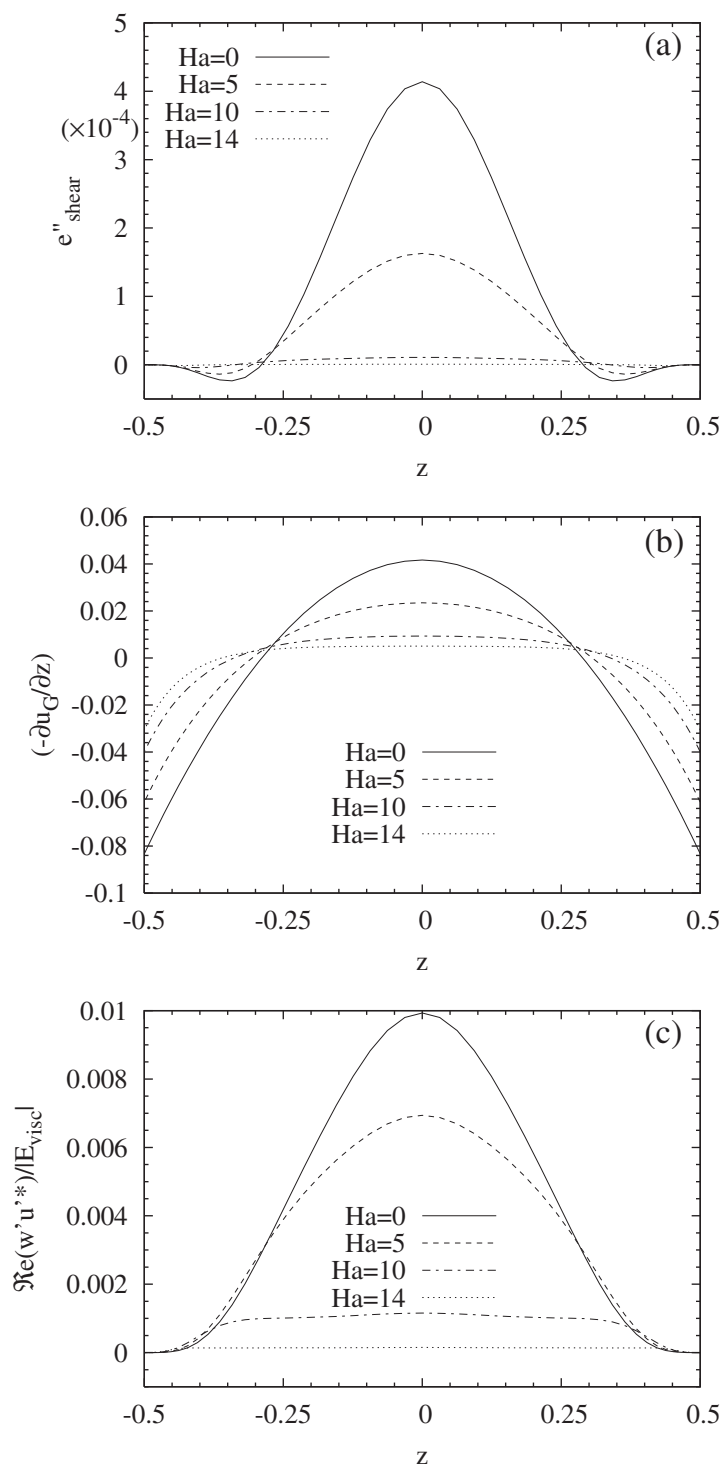

FIG. 5. Variation with $\mathrm{Ha}$ of the z-profiles of shear $e_{\text {shear }}^{\prime \prime}$ (a) and of its decomposition terms $\left(-\partial u_{G} / \partial z\right)(\mathrm{b})$ and $\left[\mathcal{R} e\left(w^{\prime} u^{\prime *}\right) /\left|E_{\mathrm{visc}}\right|\right]$ (c) associated with the basic flow and the two-dimensional steady disturbances at threshold, when a laterally heated layer at $\operatorname{Pr}=0.001$ is stabilized by a vertical magnetic field. For Ha varying from 0 to 14 , the maximum values of the profiles are, respectively, $4.1383 \times 10^{-4}, 1.6285 \times 10^{-4}, 0.1078 \times 10^{-4}$, and $0.0076 \times 10^{-4}$ for (a), $0.0417,0.0235,0.0093$, and 0.0050 for (b), 9.932 $\times 10^{-3}, 6.938 \times 10^{-3}, 1.156 \times 10^{-3}$, and $0.151 \times 10^{-3}$ for $(\mathrm{c})$

The scaled norms of the velocity disturbances contributing to the disturbance term discussed above, $\left|u^{\prime}\right| / \sqrt{\left|E_{\mathrm{visc}}\right|}$ and $\left|w^{\prime}\right| / \sqrt{\left|E_{\mathrm{visc}}\right|}$, are in turn plotted individually in Fig. 6. Both velocity components decrease strongly in the center of the layer where destabilization by shear occurs, whereas $\left|u^{\prime}\right| / \sqrt{\left|E_{\text {visc }}\right|}$ exhibit a weaker decrease along the walls, leading to the development of sharp gradients in the boundary layers of the form $\left(\partial u^{\prime} / \partial z\right)$. Moreover, these gradients increasingly dominate viscous dissipation as $\mathrm{Ha}$ increases, with contributions of $77.8 \%, 84.6 \%, 97.6 \%$, and $99.6 \%$ for $\mathrm{Ha}=0,5,10$, and 14, respectively. Thus, it is the development of these gradients as $\mathrm{Ha}$ is increased, which is responsible for the strong viscous dissipation in the Hartmann layers shown in Fig. 4(b) for $\mathrm{Ha}=14$.

Overall, the increasing contrast between the strong ve-

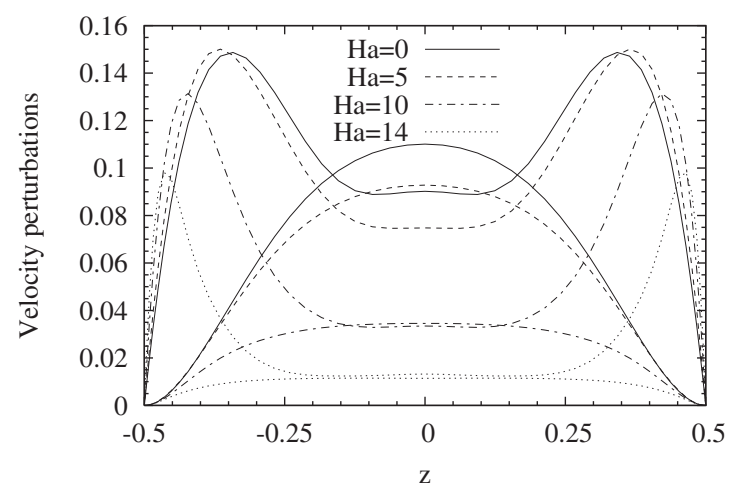

FIG. 6. Variation with $\mathrm{Ha}$ of the z-profiles of the scaled perturbations $\left|u^{\prime}\right| / \sqrt{\left|E_{\mathrm{visc}}\right|}$ (maximal along the boundaries) and $\left|w^{\prime}\right| / \sqrt{\left|E_{\mathrm{visc}}\right|}$ (maximal in the bulk) associated with the two-dimensional steady disturbances at threshold, when a laterally heated layer at $\operatorname{Pr}=0.001$ is stabilized by a vertical magnetic field.

locity gradients near the boundaries (driving the viscous dissipation energy $\left.\left|E_{\mathrm{visc}}\right|\right)$, and the weak velocities in the center of the layer [contained in $\mathcal{R} e\left(w^{\prime} u^{\prime *}\right)$ and responsible for the destabilization], seems to be at the origin of the strong decrease of $\left[\mathcal{R} e\left(w^{\prime} u^{\prime *}\right) /\left|E_{\mathrm{visc}}\right|\right]$ observed when $\mathrm{Ha}$ is increased.

In the three-dimensional cavity, however, the bulk flow is not simply proportional to Gr. Thus, we cannot extract $\mathrm{Gr}$ from the fluctuating kinetic energy equation and have to keep $E_{\text {shear }}^{\prime}$ in Eq. (8). In order to make a parallel between the simpler case of the extended layer and the three-dimensional model, the $z$-profiles of $e_{\text {shear }}^{\prime}$ and $(-\partial u / \partial z)$ at $\mathrm{Gr}_{c}$ are presented in Fig. 7 for the extended layer. [In the following, the shear $(-\partial u / \partial z)$ at $\mathrm{Gr}_{c}$ will be denoted as $(-\partial u / \partial z)_{c}$.] When Ha increases, the destabilization region indicated by the positive values of $e_{\text {shear }}^{\prime}$ broadens and the maximum value of $e_{\text {shear }}^{\prime}$ undergoes a small increase [Fig. $7(\mathrm{a})]$. The term $(-\partial u / \partial z)_{c}$ increases strongly with $\mathrm{Ha}$, but this is due to the sharp rise in $\mathrm{Gr}_{c}$ [Fig. 7(b)]. We will comment further on these profiles when discussing the three-dimensional case in Sec. III C.

\section{Shear energy analysis at threshold in the three-dimensional cavity}

A shear energy analysis analogous to that presented in Sec. III B is performed at the onset of time-dependence in the three-dimensional cavity. We focus on the dominant destabilizing shear term connected to $(\partial u / \partial z)$ and analyze its evolution with increasing magnetic field. As in Sec. III B, this shear energy term is the volume integral of the product of two terms evaluated at threshold, the derivative of the basic flow, $(-\partial u / \partial z)_{c}$, and the product of the velocity fluctuations divided by the viscous dissipation term, $\left[\mathcal{R} e\left(w^{\prime} u^{\prime *}\right) /\left|E_{\mathrm{visc}}\right|\right]$.

Isolines of these two fields and their product are plotted in the $H_{l}$ and $V_{t}$ planes (see Fig. 1 for the definition of the planes) in Fig. 8 for $\mathrm{Ha}=0$. The symmetries of the bulk flow $\left(S_{r}\right.$ and $\left.S_{l}\right)$ are both broken at the Hopf bifurcation point, but the flow retains its symmetry about the center of the cavity, $S_{c}$ (see Sec. II). Because of the $S_{l}$ symmetry breaking, the velocity fluctuations $u^{\prime}$ and $w^{\prime}$ have opposite signs at points 

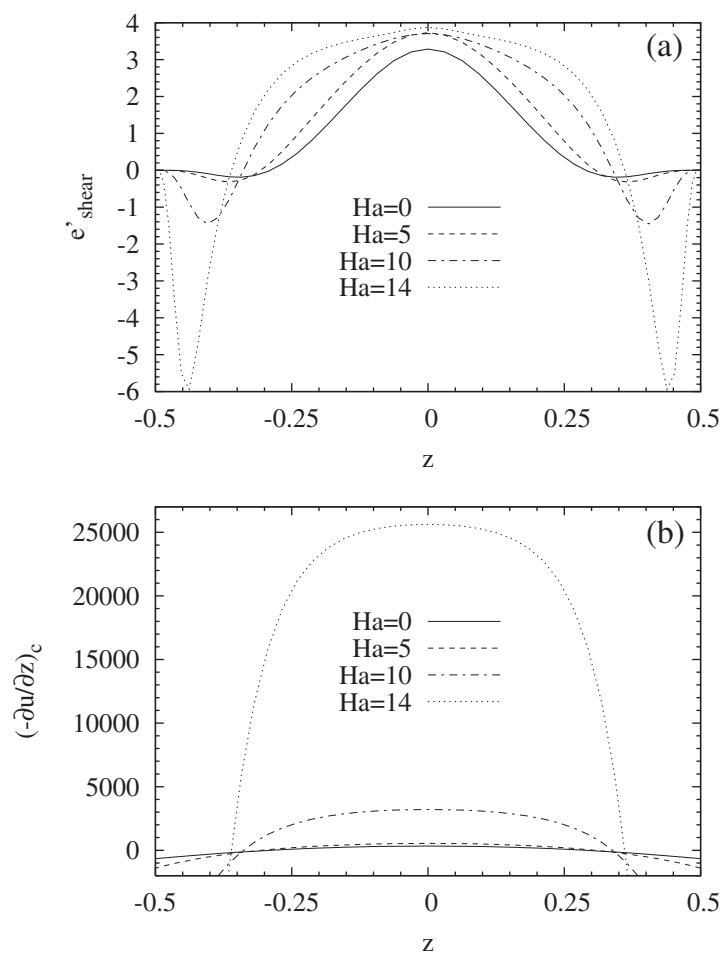

FIG. 7. Variation with Ha of the $z$-profiles of shear $e_{\text {shear }}^{\prime}$ (a) and of one of its decomposition terms $(-\partial u / \partial z)_{c}$ (b) the other term $\left[\mathcal{R} e\left(w^{\prime} u^{\prime *}\right) /\left|E_{\mathrm{visc}}\right|\right]$ is already given in Fig. 5(c)\} associated with the basic flow and the twodimensional steady disturbances at threshold, when a laterally heated layer at $\operatorname{Pr}=0.001$ is stabilized by a vertical magnetic field. For clarity, the plot of $(-\partial u / \partial z)_{c}$ is focused on the positive values. For Ha varying from 0 to 14 , the maximum values of the profiles are, respectively, 3.287, 3.731, 3.705, and 3.873 for (a), 331, 538, 3205, and 25629 for (b).

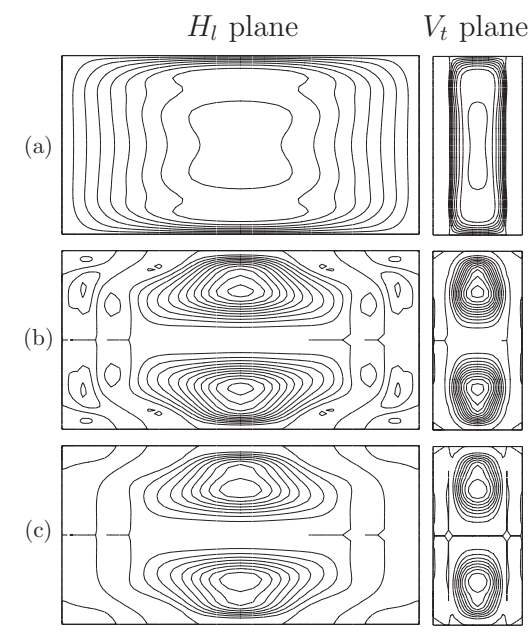

FIG. 8. Isolines of $(-\partial u / \partial z)_{c}$ (a), $\left[\mathcal{R} e\left(w^{\prime} u^{\prime *}\right) /\left|E_{\mathrm{visc}}\right|\right]$ (b), and $(-\partial u / \partial z)_{c}\left[\mathcal{R} e\left(w^{\prime} u^{\prime *}\right) /\left|E_{\mathrm{visc}}\right|\right]$ (c) associated with the basic flow and the oscillatory disturbances at $\mathrm{Gr}_{c}$ in a laterally heated three-dimensional cavity without magnetic field $(\mathrm{Ha}=0)$ : views in the $H_{l}$ plane (left pictures) and in the $V_{t}$ plane (right pictures). For $(-\partial u / \partial z)_{c}$, nine isolines are plotted from 0 to 800 (step 100; 0 is on the vertical boundaries and on the isolines intersecting these boundaries; for clarity, the negative isolines in the $V_{t}$ plane are not given); for $\left[\mathcal{R} e\left(w^{\prime} u^{\prime *}\right) /\left|E_{\mathrm{visc}}\right|\right], 14$ isolines from $-0.6 \times 10^{-3}$ to 3.3 $\times 10^{-3}$ (step $0.3 \times 10^{-3}$; 0 is on the boundaries and on the isolines intersecting the boundaries); for $(-\partial u / \partial z)_{c}\left[\mathcal{R} e\left(w^{\prime} u^{\prime *}\right) /\left|E_{\text {visc }}\right|\right]$, nine isolines from 0 to 2.4 (step 0.3 ; 0 is on the boundaries and on the isolines intersecting the boundaries). Other parameters are $A_{x}=4, A_{y}=2$, and $\operatorname{Pr}=0.026$.
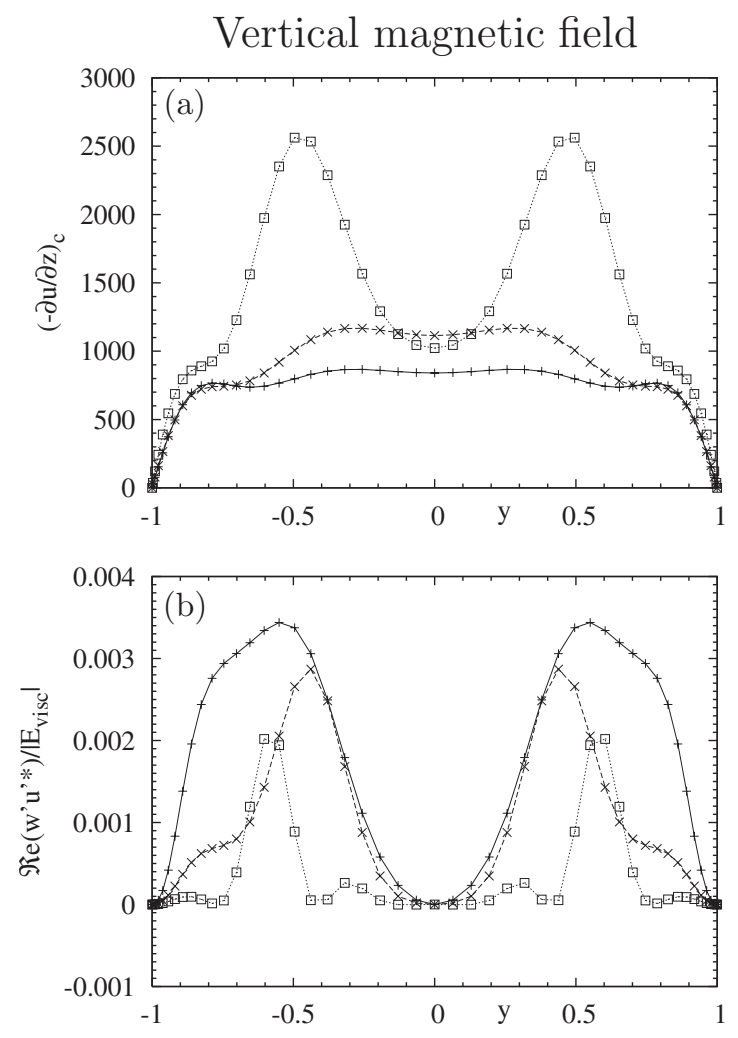

FIG. 9. Variation with $\mathrm{Ha}$ of the $y$-profiles of $(-\partial u / \partial z)_{c}$ (a) and $\left[\mathcal{R} e\left(w^{\prime} u^{\prime} *\right) /\left|E_{\mathrm{visc}}\right|\right]$ (b) associated with the basic flow and the oscillatory disturbances at $\mathrm{Gr}_{c}$ in a laterally heated three-dimensional cavity submitted to a vertical magnetic field $(\mathrm{Ha}=0,5$, and 8 labelled by,$+ \times, \square$, respectively). Other parameters are $A_{x}=4, A_{y}=2$, and $\operatorname{Pr}=0.026$.

reflected about the $V_{l}$ plane and are zero in this plane. The breaking of the $S_{r}$ symmetry results in $u^{\prime}$ and $w^{\prime}$ retaining the same sign at points separated by a $\pi$-rotation about the transverse $y$-axis. Thus, the product of $u^{\prime}$ and $w^{\prime}$ retains the same sign at all these points, and is zero in the $V_{l}$ plane. In Fig. 8(b), [Re( $\left.\left.w^{\prime} u^{\prime *}\right) /\left|E_{\mathrm{visc}}\right|\right]$ takes significant positive values in two regions at midheight and midlength in the cavity on either side of the $V_{l}$ plane. $(-\partial u / \partial z)_{c}$ remains positive and approximately constant in these regions [see Fig. 8(a)]. Hence, it is the velocity disturbance field which primarily determines the domain where $(-\partial u / \partial z)_{c}\left[\mathcal{R} e\left(w^{\prime} u^{\prime *}\right) /\left|E_{\mathrm{visc}}\right|\right]$ has non-negligible positive values [Fig. 8(c)].

The same scalar fields as in Fig. 8 were examined at threshold when vertical, transverse and longitudinal magnetic fields were applied. The regions where the contribution of the shear energy is not negligible are similar to those observed in the case of $\mathrm{Ha}=0$. Similar general trends were also found in all three cases, with an increase of $(-\partial u / \partial z)_{c}$ and a decrease of $\left[\mathcal{R} e\left(w^{\prime} u^{\prime *}\right) /\left|E_{\mathrm{visc}}\right|\right]$, as Ha is raised. These findings are analogous to the results in the extended layer [see Figs. 7(b) and 5(c)].

A quantitative measure of the effect of the magnetic field on the local components of the shear energy at threshold is obtained by plotting profiles of $(-\partial u / \partial z)_{c}$ and $\left[\mathcal{R} e\left(w^{\prime} u^{\prime *}\right) /\left|E_{\mathrm{visc}}\right|\right]$ along the $y$ axis $(z=0$ and $x=0)$. The profiles are shown in Figs. 9-11 for the vertical, transverse, and longitudinal directions of the magnetic field, respectively. A reduction in $\left[\mathcal{R} e\left(w^{\prime} u^{\prime *}\right) /\left|E_{\mathrm{visc}}\right|\right]$ occurs as $\mathrm{Ha}$ is 


\section{Transverse magnetic field}
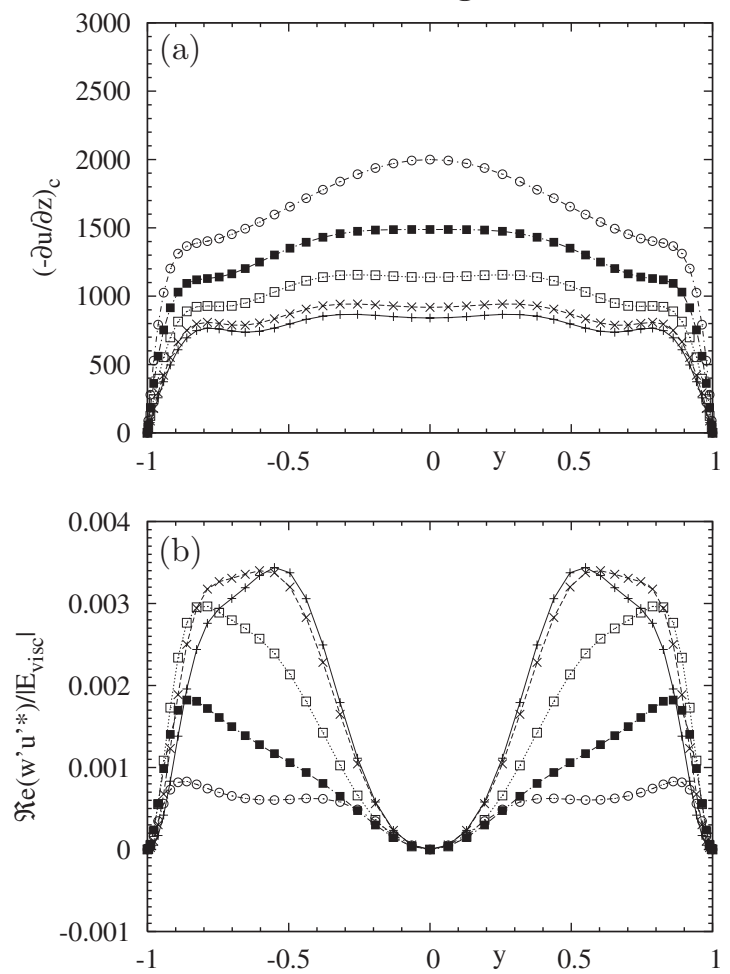

FIG. 10. Variation with $\mathrm{Ha}$ of the $y$-profiles of $(-\partial u / \partial z)_{c}$ (a) and $\left[\mathcal{R} e\left(w^{\prime} u^{\prime *}\right) /\left|E_{\mathrm{visc}}\right|\right]$ (b) associated with the basic flow and the oscillatory disturbances at $\mathrm{Gr}_{c}$ in a laterally heated three-dimensional cavity submitted to a transverse magnetic field $(\mathrm{Ha}=0,5,10,15$, and 20 labelled by,$+ \times, \square$,

$\mathbf{\square}, \bigcirc$, respectively). Other parameters are $A_{x}=4, A_{y}=2$, and $\operatorname{Pr}=0.026$.

increased in all three cases, which is similar to, albeit weaker than, that observed in the case of the extended layer [see Fig. 5(c)]. This decrease is accompanied by an expansion of the profiles in the longitudinal direction, and in the case of the vertical magnetic field, by a narrowing of the profiles in the transverse direction, which further contributes to the decrease of the $\left[\mathcal{R} e\left(w^{\prime} u^{\prime *}\right) /\left|E_{\mathrm{visc}}\right|\right]$ contribution to the shear energy term. In order to retain the energy balance necessary to trigger the onset of time-dependence, the reduction in $\left[\mathcal{R} e\left(w^{\prime} u^{\prime *}\right) /\left|E_{\mathrm{visc}}\right|\right]$ induces a strong increase of $(-\partial u / \partial z)_{c}$ in all three cases, but this is most pronounced in the case of the vertical field, where it occurs for small values of Ha. [The appearance of peaks in the $y$-profile of $(-\partial u / \partial z)_{c}$ in the presence of a vertical magnetic field is linked to the preferential development of the longitudinal basic velocity in the parallel layers as Ha is increased. $\left.{ }^{9}\right]$ Moreover, the increase of $(-\partial u / \partial z)_{c}$ in the presence of transverse and longitudinal fields is additionally linked to the global increase of the shear energy term connected to $(-\partial u / \partial z)\left[\mathcal{R} e\left(w^{\prime} u^{\prime *}\right) /\left|E_{\mathrm{visc}}\right|\right]$, which occurs in order to compensate for the stabilizing effect of the magnetic energy, as discussed in Sec. III A. Note that this stabilizing effect becomes significant at lower values of $\mathrm{Ha}$ for the transverse magnetic field than for the longitudinal field (see Fig. 3).

It is the growth with $\mathrm{Ha}$ of the term $(-\partial u / \partial z)_{c}$ that explains the rise in critical Grashof number. A similar, albeit stronger, increase of $(-\partial u / \partial z)_{c}$ was also identified in the extended layer [see Fig. 7(b)]. In that configuration, however,
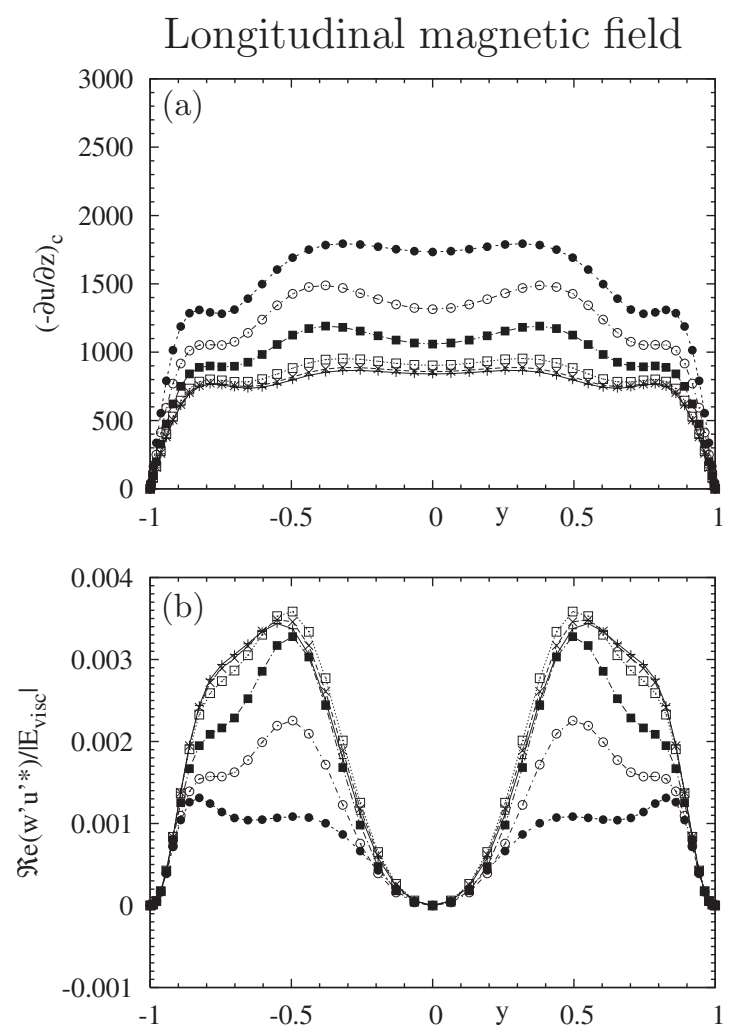

FIG. 11. Variation with $\mathrm{Ha}$ of the $y$-profiles of $(-\partial u / \partial z)_{c}$ (a) and $\left[\mathcal{R} e\left(w^{\prime} u^{\prime *}\right) /\left|E_{\mathrm{visc}}\right|\right]$ (b) associated with the basic flow and the oscillatory disturbances at $\mathrm{Gr}_{c}$ in a laterally heated three-dimensional cavity submitted to a longitudinal magnetic field $(\mathrm{Ha}=0,5,10,20,30$, and 40 labelled by + , $\times, \square, \mathbf{\square}, \bigcirc$, ○, respectively). Other parameters are $A_{x}=4, A_{y}=2$, and $\operatorname{Pr}$ $=0.026$.

the situation was simpler to analyze because of the proportionality of the basic velocity field with $\mathrm{Gr}$, which allowed us to define $\left(-\partial u_{G} / \partial z\right)$ independent of $\mathrm{Gr}_{c}$ and depending only on Ha [see Fig. 5(b)]. The strong increase of $\mathrm{Gr}_{c}$ with Ha (by a factor of 640.6 when Ha was varied from 0 to 14 in Table II) might be justified by the combined effects of the strong increase of $(-\partial u / \partial z)_{c}$ \{maximum value multiplied by 77.43 [Fig. 7(b)]\} and the decrease of $\left(-\partial u_{G} / \partial z\right)$ \{maximum value divided by 8.34 [Fig. 5(b)]\}. In the case of the threedimensional cavity, a similar exact analysis cannot be performed because the influences of $\mathrm{Gr}$ and $\mathrm{Ha}$ on the basic velocity field cannot be isolated. The effect of $\mathrm{Ha}$ on the basic velocity field, however, can be studied at a fixed value of Gr. For $\mathrm{Gr}=G r_{c_{0}}$ (the threshold value for $\mathrm{Ha}=0$ ), the maximum values of $(-\partial u / \partial z)$ are shown to decrease with increasing $\mathrm{Ha}$ in Fig. 12 for the three directions of the magnetic field. This evidence suggests that the increase of $\mathrm{Gr}_{c}$ with Ha must be particularly strong to induce the observed increase of $(-\partial u / \partial z)_{c}$ at $\mathrm{Gr}_{c}$, when at constant $\mathrm{Gr}=G r_{c_{0}}$, this term would decay with increasing Ha. The reduction is particularly steep in the case of the vertical magnetic field, consistently with the observation of the strongest increase of $\mathrm{Gr}_{c}$, whereas the decay of $(-\partial u / \partial z)$ at constant $\mathrm{Gr}$ for the transverse and longitudinal fields is weaker and postponed to larger values of $\mathrm{Ha}$ (a slight increase is even observed for the transverse field for Ha between 5 and 10).

The results presented in this section demonstrate that for 


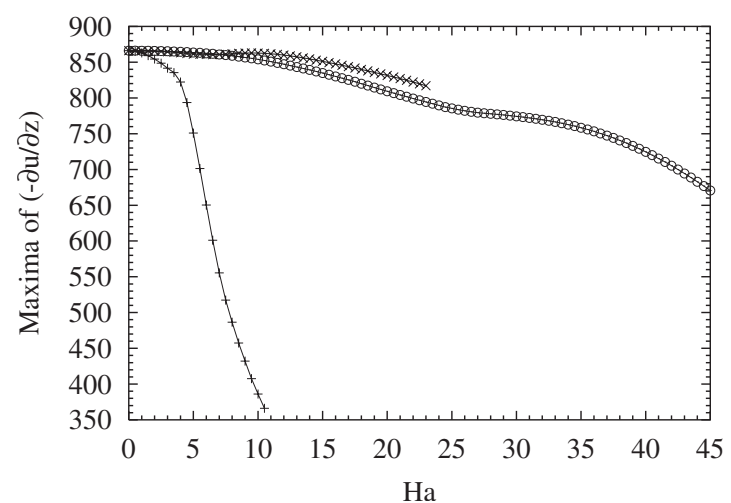

FIG. 12. Variation of $(-\partial u / \partial z)_{\max }$ as a function of $\mathrm{Ha}$ at constant $\mathrm{Gr}$ $=G r_{c_{0}}$ (threshold without magnetic field) for different orientations of the magnetic field (+ for the vertical magnetic field, $\times$ for the transverse field, $\bigcirc$ for the longitudinal field). Other parameters are $A_{x}=4, A_{y}=2$, and $\operatorname{Pr}$ $=0.026$.

a vertical magnetic field, both the strong decrease of $\left[\mathcal{R} e\left(w^{\prime} u^{\prime *}\right) /\left|E_{\mathrm{visc}}\right|\right]$ (decrease of its intensity and narrowing of the productive zone) and the strong decrease of $(-\partial u / \partial z)$ at constant $G r$ with increasing Ha, are responsible for the sharp rise in the thresholds, while the influence of the magnetic dissipation is found to be weak. For the transverse and longitudinal magnetic fields, however, the magnetic dissipation is significant as it induces an increase of the dominant shear term. It is nevertheless the decrease of $\left[\mathcal{R} e\left(w^{\prime} u^{\prime *}\right) /\left|E_{\mathrm{visc}}\right|\right]$, although less efficient than for the vertical field, but more efficient for the transverse field than for the longitudinal field, which dominates the increase of the thresholds with Ha.

Finally, the analysis performed on the extended layer model suggests that the decrease of $\left[\mathcal{R} e\left(w^{\prime} u^{\prime *}\right) /\left|E_{\mathrm{visc}}\right|\right]$ as $\mathrm{Ha}$ is increased may be linked to the combined effects of strong gradients in the velocity fluctuations developing in the Hartmann boundary layers along the walls (these gradients induce stronger viscous dissipation), and comparatively weak velocity fluctuations in the bulk where the destabilization process by shear is effective.

\section{CONCLUSION}

The directional effect of a magnetic field on the onset of time-periodic convection has been studied numerically in a confined three-dimensional cavity. The critical Grashof number and frequency at the Hopf bifurcation point exhibit similar exponential dependencies on the Hartmann number, Ha. The vertical field is the most efficient at postponing the onset of oscillations to larger values of Gr, followed by the transverse and longitudinal fields, in accordance with the experimental findings of Hof et al. ${ }^{7}$

The variation of the global energy budget with Ha, calculated at threshold for each of the three principal directions of the magnetic field, indicates that the oscillatory transition is dominated by the vertical shear of the longitudinal flow, and that the magnetic energy is not the dominant source of stabilization, particularly in the presence of a vertical magnetic field. The examination of the spatial distribution of the dominant shear energy term is required to gain insight into the magnetohydrodynamic damping mechanism. This quantity is given by the product of the shear of the basic flow $(-\partial u / \partial z)_{c}$ at $\mathrm{Gr}_{c}$ and the velocity fluctuations $\left\{\left[\mathcal{R} e\left(w^{\prime} u^{\prime *}\right) /\left|E_{\mathrm{visc}}\right|\right]\right\}$. The strong decrease of $\left(\mathcal{R} e\left(w^{\prime} u^{\prime *}\right) /\left|E_{\mathrm{visc}}\right|\right)$ that results from the formation of steep gradients of the velocity fluctuations in the Hartmann layers and the weakening of these velocity fluctuations in the bulk, couples to the decrease of $(-\partial u / \partial z)$ at constant Gr with increasing Ha to induce the exponential growth in critical parameters. This mechanism alone is at the origin of the damping in the case of a vertical magnetic field, whereas for transverse and longitudinal fields, it acts in combination with the growth in stabilizing magnetic energy.

\section{ACKNOWLEDGMENTS}

This work was funded by an Alliance Partnership grant from the British Council and Egide (A.J. and D.H.) and an EPSRC Advanced Research Fellowship (A.J.). The calculations were carried out on a NEC-SX5 computer with the support of the CNRS through the Institut du Développement et des Ressources en Informatique Scientifique.

${ }^{1}$ G. Müller and A. Ostrogorsky, "Convection in melt growth," in Handbook of Crystal Growth: Growth Mechanisms and Dynamics, edited by D. T. J. Hurle (North-Holland, Amsterdam, 1993), Vol. 2b.

${ }^{2}$ A. Müller and M. Wiehelm, "Periodische temperaturschwankungen in flüssigem InSb als ursache schichtweisen einbaus von Te in kristallisierendes InSb," Z. Naturforsch. A 19, 254 (1964).

${ }^{3}$ H. P. Utech and M. C. Flemings, "Elimination of solute banding in indium antimonide crystals by growth in a magnetic field," J. Appl. Phys. 37, 2021 (1966).

${ }^{4}$ D. T. J. Hurle, "Temperature oscillations in molten metals and their relationship to growth striae in melt-grown crystals," Philos. Mag. 13, 305 (1966).

${ }^{5}$ D. T. J. Hurle, E. Jakeman, and C. P. Johnson, "Convective temperature oscillations in molten gallium,” J. Fluid Mech. 64, 565 (1974).

${ }^{6}$ K. E. McKell, D. S. Broomhead, R. Jones, and D. T. J. Hurle, "Torus doubling in convecting molten gallium," Europhys. Lett. 12, 513 (1990).

${ }^{7}$ B. Hof, A. Juel, and T. Mullin, "Magnetohydrodynamic damping of oscillations in low-Prandtl-number convection," J. Fluid Mech. 545, 193 (2005).

${ }^{8}$ H. Ben Hadid and D. Henry, "Numerical simulation of convective threedimensional flows in a horizontal Bridgman configuration under the action of a constant magnetic field," in Proceedings of the Second International Conference on Energy Transfer in Magnetohydrodynamic Flows, Aussois, France (MHD Pamir, Grenoble, 1994), Vol. 1, pp. 47-56.

${ }^{9}$ H. Ben Hadid and D. Henry, "Numerical study of convection in the horizontal Bridgman configuration under the action of a constant magnetic field. Part 2: Three-dimensional flow," J. Fluid Mech. 333, 57 (1997).

${ }^{10}$ H. Ben Hadid and D. Henry, "Numerical simulations of convective threedimensional flows in a horizontal cylinder under the action of a constant magnetic field," J. Cryst. Growth 166, 436 (1996).

${ }^{11}$ A. Juel, T. Mullin, H. Ben Hadid, and D. Henry, "Magnetohydrodynamic convection in molten gallium," J. Fluid Mech. 378, 97 (1999).

${ }^{12}$ B. Hof, A. Juel, and T. Mullin, "Magnetohydrodynamic damping of convective flows in molten gallium," J. Fluid Mech. 482, 163 (2003).

${ }^{13}$ D. Henry and H. Ben Hadid, "Multiple flow transitions in a box heated from the side in low-Prandtl-number fluids," Phys. Rev. E 76, 016314 (2007).

${ }^{14}$ D. Henry and M. Buffat, "Two and three-dimensional numerical simulations of the transition to oscillatory convection in low-Prandtl number fluids," J. Fluid Mech. 374, 145 (1998).

${ }^{15}$ B. Hof, A. Juel, L. Zhao, D. Henry, H. Ben Hadid, and T. Mullin, "On the onset of oscillatory convection in molten gallium," J. Fluid Mech. 515, 391 (2004).

${ }^{16}$ S. Kaddeche, D. Henry, and H. Ben Hadid, "Magnetic stabilization of the 
buoyant convection between infinite horizontal walls with a horizontal temperature gradient," J. Fluid Mech. 480, 185 (2003).

${ }^{17}$ H. Ben Hadid, D. Henry, and S. Kaddeche, "Numerical study of convection in the horizontal Bridgman configuration under the action of a constant magnetic field. Part 1: Two-dimensional flow," J. Fluid Mech. 333, 23 (1997).

${ }^{18}$ T. Alboussière, D. Henry, and S. Kaddeche, "Note on braking and stabilization laws for buoyant flows under a weak magnetic field," Fluid Dyn. Res. 13, 287 (2003).

${ }^{19}$ V. Bojarevics, "Buoyancy-driven flow and its stability in a horizontal rectangular channel with an arbitrary oriented transversal magnetic field," Magnetohydrodynamics (N.Y.) 31, 245 (1995).

${ }^{20} \mathrm{~J}$. Priede and G. Gerbeth, "Hydrothermal wave instability of thermocapillary-driven convection in a coplanar magnetic field," J. Fluid Mech. 347, 141 (1997).

${ }^{21}$ J. Priede and G. Gerbeth, "Hydrothermal wave instability of thermocapillary-driven convection in a transverse magnetic field," J. Fluid Mech. 404, 211 (2000).

${ }^{22}$ A. Gelfgat and P. Bar-Yoseph, "The effect of an external magnetic field on oscillatory instability of convective flows in a rectangular cavity," Phys. Fluids 13, 2269 (2001).

${ }^{23}$ A. Juel, T. Mullin, H. Ben Hadid, and D. Henry, "Three-dimensional free convection in molten gallium," J. Fluid Mech. 436, 267 (2001).

${ }^{24}$ R. Moreau, Magnetohydrodynamics (Kluwer, Dordrecht, 1990).

${ }^{25}$ G. E. Karniadakis, M. Israeli, and S. A. Orszag, "High-order splitting method for the incompressible Navier-Stokes equations," J. Comput. Phys. 97, 414 (1991)

${ }^{26}$ R. B. Lehoucq, D. C. Sorensen, and C. Yang, ARPACK Users' Guide: Solution of Large-Scale Eigenvalue Problems with Implicitly Restarted Arnoldi Methods (SIAM, Philadelphia, 1998).

${ }^{27}$ C. K. Mamun and L. S. Tuckerman, "Asymmetry and Hopf bifurcation in spherical Couette flow," Phys. Fluids 7, 80 (1995).

${ }^{28}$ G. Petrone, E. Chénier, and G. Lauriat, "Stability of free convection in air-filled horizontal annuli: Influence of the radius ratio," Int. J. Heat Mass Transfer 47, 3889 (2004).

${ }^{29}$ D. R. Kincaid, T. C. Oppe, and W. D. Joubert, “An overview of NSPCG: A nonsymmetric preconditioned conjugate gradient package," Comput. Phys. Commun. 53, 283 (1989). 